\title{
The relationship between color-object associations and color preference: Further investigation of ecological valence theory
}

\author{
Chloe Taylor • Anna Franklin
}

Published online: 3 February 2012

(C) Psychonomic Society, Inc. 2012

\begin{abstract}
Ecological valence theory (EVT; Palmer \& Schloss, Proceedings of the National Academy of Sciences $107: 8877-8882,2010)$ proposes that color preferences are due to affective responses to color-associated objects: That is, people generally like colors to the degree that they like the objects associated with those colors. Palmer and Schloss found that the average valence of objects associated with a color, when weighted by how well the objects matched the color (weighted affective valence estimates: WAVE) explained $80 \%$ of the variation in preference across colors. Here, we replicated and extended Palmer and Schloss's investigation to establish whether color-object associations can account for sex differences in color preference and whether the relationship between associated objects and color preference is equally strong for males and females. We found some degree of sex specificity to the WAVEs, but the relationship between WAVE and color preference was significantly stronger for males than for females $(74 \%$ shared variance for males, $45 \%$ for females). Furthermore, analyses identified a significant inverse relationship between the number of objects associated with a color and preference for the color. Participants generally liked colors associated with few objects and disliked colors associated with many objects. For the sample overall and for females alone, this association was not significantly weaker than the association of the WAVE and preference. The success of the WAVE at
\end{abstract}

Electronic supplementary material The online version of this article (doi:10.3758/s13423-012-0222-1) contains supplementary material, which is available to authorized users.

C. Taylor

Department of Psychology, University of Surrey,

Guildford, Surrey GU2 5XH, UK

A. Franklin $(\bowtie)$

School of Psychology, University of Sussex,

Brighton, Sussex BN1 9RH, UK

e-mail: anna.franklin@sussex.ac.uk capturing color preference was partly due to the relationship between the number of associated objects and color preference. The findings identify constraints of EVT in its current form, but they also provide general support for the link between color preference and color-object associations.

Keywords Visual perception · Color P Preference

Although individuals vary in which colors they like and dislike, research has established that common, systematic patterns of color preference occur within and across cultures and genders (e.g., Hurlbert \& Ling, 2007; Ou, Luo, Woodcock, \& Wright, 2004; Saito, 1996). The current challenge for researchers is to establish why some colors are more liked than others. Why are there affective responses to color, given that it is a basic visual stimulus? What is it about color that "holds" affect (Zajonc, 1980)? A common theme in theories of color preference is that commonality in color preference (e.g., the common preference for blue and aversion to yellow) results from preferences being largely "hardwired" or biologically determined (e.g., Humphrey, 1976; Hurlbert \& Ling, 2007; Walton, Guilford, \& Guilford, 1933). For example, in one recent theory it was proposed that color preference is governed by the two neural subsystems that underlie human color vision (the "red-green" L-M and "blue-yellow" S-(L+M) cone-opponent processes), and that a "universal" sex difference in the weighting of the "redgreen" biological component of color preference evolved in line with sex differences in the behavioral use of color vision (Hurlbert \& Ling, 2007). However, given the variation in color preference across individuals and cultures, a purely biological model of color preference is unable to fully explain color preference. For example, in Hurlbert and Ling's model, individuals and cultures can weight the biological components of color preference 
differently, yet nonbiological factors are required in order to explain why.

Another recent theory of color preference, the "ecological valence theory" (EVT), has attempted to explain both the commonality and individuality of color preference (Palmer \& Schloss, 2010). Palmer and Schloss proposed that color preferences result from affective responses to colorassociated objects. In other words, we like or dislike colors to the degree that they are associated with liked or disliked objects. EVT suggests that this association has an ecological and adaptive function: It is good for survival if colors that are associated with good objects (e.g., clean water) are preferred and colors associated with objects that we should avoid (e.g., rotten food) are disliked. EVT also proposes that color preferences can be modified within an individual's lifetime by their experience of, and affective response to, objects. The theory has the potential to explain the commonality in color preference (some common objects will be liked by all of us, such as clean water) and to explain the variation in color preference (different individuals, genders or cultures experience different objects and differ in their affective responses to objects).

Palmer and Schloss (2010) provided support for EVT by showing that the average valences of color-associated objects are strongly associated with color preference. A sample of American participants rated their preference for a set of colors; different samples listed objects associated with those colors, how much they liked the associated objects, and how well the associated objects matched the given color.

These object measures were then used to obtain a "weighted affective valence estimate" (WAVE) for each color. The WAVE weights the object valence ratings by how well the object is judged to match the color, and then averages all of the weighted valences (by dividing the sum of the weighted valences for a color by the number of associated objects). The resulting WAVE bore a striking resemblance to the color preference curves: Both had peaks in preference around blue, minima at dark yellow, and $80 \%$ of the variance in the color preference curves and the WAVE was shared.

Palmer and Schloss (2010) also reported ongoing investigations of whether the EVT can account for variation in preference across cultures. Preliminary results from Japan indicated that color preference and the WAVE for Japanese participants shared considerably less variance (44\%) than did the association for the American sample. However, both Japanese and American WAVEs shared more variance with the color preferences of the corresponding culture, indicating some degree of cultural specificity of the relationship between the WAVE and preference. Further evidence that color preferences are influenced by a group's experience of objects or entities comes from a study of the color preferences of Berkeley and Stanford students (Schloss, Poggesi, \& Palmer, 2011). Participants preferred their university colors (e.g., colors used for sports teams and marketing) over the other university's colors, and this effect was stronger the more that students liked and identified with their university.

In the present study, we replicated and extended Palmer and Schloss's original (2010) investigation, to establish whether EVT can account for both male and female color preferences. Gender differences extend beyond the idiom "pink is for girls, blue is for boys" (e.g., LoBue \& DeLoache, in press); for example, studies have reported sex differences in the preference for orange (e.g., Walton et al., 1933) and greenish (e.g., Hurlbert \& Ling, 2007) hues. EVT predicts that sex differences such as these can be accounted for by sex differences in the objects associated with the colors and in those objects' valences. This prediction could not be tested in Palmer and Schloss's investigation, as object associations were elicited in groups, and therefore separate object lists for males and females could not be drawn up. In the present study, to assess the relationship between WAVEs and male and female color preference, individuals, rather than groups, listed objects associated with the colors, enabling us to compute separate WAVEs for males and females.

In addition, we further explored the relationship between object associations and color preference by investigating whether the number of objects associated with a color is related to preference for that color. From our own observations, we noted that it is difficult to list many objects that are associated with blue, a commonly preferred color, but easier to list objects that are brown, a generally disliked color. Our secondary analysis of Palmer and Schloss's (2010) object descriptions (see their Table S2) subsequently revealed a significant negative correlation between color preference and the number of objects associated with that color $(r=$ $-.66, p<.001)$. The number of objects associated with a color does not directly assess valence, and therefore this relationship could suggest that the influence of color-object associations is not restricted to object valence ratings. To investigate this, we further assessed the relationship between the number of objects associated with a given color and color preference. As the number of objects is part of the WAVE formula, we also assessed its contribution to the preference-WAVE association. Palmer and Schloss's investigation had a time limit for groups to list objects associated with a color (20 s), but here we gave participants no explicit time limit, in order that there would be no restriction on how many objects could be listed (see the Method section).

\section{Method}

\section{Participants}

There were different samples for each task, as in Palmer and Schloss (2010): for color preference, 22 males (mean age $=$ 
24 years, $S D=6.60$ ) and 20 females (mean age $=21$ years, $S D=6.82$ ); for object description, 26 males (mean age $=$ 23 years, $S D=4.92$ ) and 29 females (mean age $=20$ years, $S D=2.44$ ); for color-object matching, 18 males (mean age = 24 years, $S D=2.91$ ) and 20 females (mean age $=23$ years, $S D=3.73$ ); and for object valence, 20 males (mean age = 22 years, $S D=4.43$ ) and 20 females (mean age $=22$ years, $S D=3.38$ ). The overall mean age of the males (mean age $=$ 23 years, $S D=4.58$ ) was marginally greater than that of the females (mean age $=22$ years, $S D=4.28$ ), but not significantly so, $t(168)=1.80, p=.07$. All participants were students at the University of Surrey in England, were British citizens, and were screened for color vision deficiencies with the City Colour Vision Test (Fletcher, 1981).

\section{Stimuli and setup}

Colors were approximations of those from the "saturated" (S), "light" (L), and "dark" (D) stimulus sets in Palmer and Schloss (2010). The "saturated" set comprised eight saturated hues that were good examples of red $(\mathrm{R})$, orange $(\mathrm{O})$, yellow $(Y)$, chartreuse $(\mathrm{H})$, green $(\mathrm{G})$, cyan $(\mathrm{C})$, blue $(\mathrm{B})$, and purple (P). The "light" set had light and desaturated versions of the eight hues: Lightness and saturation values were approximately halfway between those of the corresponding color in the saturated set and Munsell Value 9 (light) and Munsell Chroma 1 (desaturated). The "dark" set had dark and desaturated versions of the eight hues: Lightness and saturation values were approximately halfway between those of the corresponding color in the saturated set and Munsell Value 1 (dark) and Munsell Chroma 1 (desaturated). The "muted" set of Palmer and Schloss, in which the eight hues were at midluminance and desaturated, was not included, as Palmer and Schloss's "muted" preference curves and WAVEs were highly similar to those of the "light" set. Table S1 (in the supplementary materials) gives the stimulus coordinates in Munsell color space and the chromaticity coordinates, verified by a colorimeter (Cambridge Research Systems ColorCal, Rochester, U.K.). Colors were presented on a calibrated 21-in. CRT monitor (Sony Trinitron GDM-F520; participants were at a distance of $57 \mathrm{~cm})$ as rectangular patches $\left(11.6^{\circ}\right.$ horizontal, $12.1^{\circ}$ vertical) in the center of the screen and on a gray background $\left(Y=17.75 \mathrm{~cd} / \mathrm{m}^{2}, x=0.312, y=0.318\right)$. Participants were tested in a dark room.

\section{Task design and procedure}

Color preference The stimuli were presented individually, and participants were asked to rate how much they liked the color $(0=$ not at all, $10=$ very much $)$. A total of 48 trials (two per stimulus) were presented in a randomized order, and a gray background was presented for $500 \mathrm{~ms}$ in between trials. Participants were asked to respond quickly and instinctively. The stimulus stayed on the screen until the participants had made their responses.

Object description task The stimuli and setup were identical to those of the color preference task, but instead of rating preference for each color, participants were asked to list objects that they associated with the color. As in Palmer and Schloss (2010), we asked participants to list only objects that were specific to the presented color and not unique to themselves (e.g., "my favorite sweater"), and not to list objects that could be a variety of colors (cars, paint, etc.) or to list abstract concepts such as "happiness." Unlike in Palmer and Schloss's study, our participants were not given an explicit time limit to list associations: The color remained on screen until participants had finished listing their associations - no one required longer than two minutes per color-and the overall task took around $30 \mathrm{~min}$ per participant. Also unlike Palmer and Schloss's procedure, we tested our participants individually rather than in groups. The stimuli were shown once in a randomized order.

Color-object matching task Following Palmer and Schloss's (2010) criteria, objects were removed from the object list if they could be any color (e.g., cars), described a concept (such as "peace," "love," etc.) instead of an object, or did not match the color on the screen at all (e.g., "banana" for saturated blue). Few associations needed to be removed, as participants generally followed the task instructions. The removed object associations were "sweets", "fondant fancies" (a type of cake that comes in many colors), and "TV," as all of these could be any color, and "royalty," "horizon," and "angry person," as these were deemed to be abstract or not clearly identifiable. ${ }^{1}$ After these exclusions, a list of 282 objects remained. Colors were presented as in the color preference task. Participants were asked to judge how well the color of the stimulus matched the color of each object associated with that color using a rating scale from 0 (very poorly) to 10 (very well). Color order and the order of objects were randomized.

\footnotetext{
${ }^{1}$ It was sometimes difficult to decide whether the object association fulfilled Palmer and Schloss's (2010) criteria for exclusion. For example, flowers come in many colors, and therefore associations such as "purple flowers" could be excluded from the list for this reason. However, here, as in Palmer and Schloss's study, we included these associations, as although flowers come in many colors, they cannot be any color. Additionally, the association with Ireland and green could on some level be classified as abstract, although the land of the country is actually green. These cases highlight an element of subjectivity in the classification of color-object associations. However, importantly, the following analyses were not affected by whether or not the above "dubious" associations were included or excluded from the data. For clarity, we highlight the dubious associations in Table S2 of the supplementary information.
} 
Object valence task Participants rated how much they liked each of the 282 objects using a valence scale $(0=$ not at all, $5=$ neutral, $10=$ very much $)$. The participants did the task at their own pace.

\section{Results}

Individuals' preference ratings for the first and second times that they rated the colors were significantly related, $r=.70$, $p<.001$. In addition, this group's preference ratings (the ratings averaged across individuals) were highly significantly related between first and second ratings, $r=.95$, $p<.001$. This indicates that participants' preference ratings were reliable, particularly the group color preferences on which the following analysis is based. Therefore, each participant's preference for each color was averaged across the two stimulus presentations, and the average color preference rating for each color was then calculated across participants and for males and females (see Fig. 1). The object valence ratings were converted to a -5 to +5 scale, with 5 (neutral) on the original scale set to $0 .^{2}$ The WAVE for each color was calculated using the Palmer and Schloss (2010) formula:

$W_{c}=\frac{1}{n_{c}} \sum_{o=1}^{n_{c}} w_{c o} v_{o}$

where $w_{c o}$ is the average color-object match value for each object ( $o$, converted to a 0 to 1 scale) and its associated color $(c), v_{o}$ is the average preference rating for each object $o$, and $n_{c}$ is the number of objects associated with each color $c$ (see Table 2 in the supplementary information for a breakdown of the data). The WAVE was calculated for the sample overall and for males and females separately by using the male or female data for the object descriptions, object-color match values, and object preference ratings.

As can be seen in Fig. 1, there are similarities between the shapes of the preference curves and the WAVEs. For example, the preference curves peak at blue, and the WAVEs also peak at blue (blue is associated with positive objects such as water and the sky). There are also notable differences between the preference curves and the WAVEs. For example, the preference minima overall and for males and females are at dark yellow, yet the WAVE minima are at dark chartreuse. Dark chartreuse is associated with many disliked objects, such as bile, mucus, mold, sewage, and snot, and with fewer liked objects, whereas dark yellow

\footnotetext{
${ }^{2}$ None of the valence ratings (averaged across participants) for any of the objects were identified as outliers when converted to $z$ scores and compared to a criterion of \pm 3.29 .
}

is associated with disliked objects such as feces, pus, and vomit, but also with liked objects such as apple juice, pineapple, and sunflowers. The preference curves are similar for males and females $(r=.66, p<.001)$, with notable exceptions, such as males' stronger preference for saturated and dark orange. This sex difference for orange is captured by the WAVE, as the male WAVE peaks at saturated orange, but the female WAVE doesn't (partly due to males but not females associating sunrise and sunshine with orange). The WAVE similarly mirrors the sex difference in preference for purples. Overall, the male WAVE appears to capture male preference better than the female WAVE captures female preference.

To further investigate the relationships between color preference and the WAVEs, a series of Pearson's correlations were conducted (see Fig. 2). There was a significant correlation of the WAVE and color preference overall $(r=.81, p<.001$, $66 \%$ shared variance), for the male WAVE and male preference $(r=.86, p<.001,74 \%)$, and for the female WAVE and female preference $(r=.67, p<.001,45 \%)$. As can be seen by comparing the preference and WAVE figures in Fig. 1, this relationship is consistent across colors and is not due to one spurious data point. The correlation between a given gender's WAVE and their color preference was significantly stronger for males than for females $(p<.005)$. The male WAVE was significantly more strongly associated with male than with female preference $(p<.001 ; r=.58, p<.005$, for the association with female preference). The female WAVE was not significantly more strongly associated with female than with male preference $(p=.19 ; r=.58, p<.005$, for the association with male preference).

\section{Number of associated objects}

The numbers of associated objects were calculated for each color for the overall sample and for males and females separately. As can be seen in Fig. 3, there appears to be an inverse relationship between the number of associated objects and color preference. For example, dark yellow is the least liked color and has the most objects associated with it (41 overall), while saturated blue is the most liked color and has the fewest objects associated with it (only 5 overall). Also, as color preference increases across hues from yellow to blue, the number of objects decreases. Overall, there was a significant negative correlation between color preference and the number of associated objects $(r=-.76, p<.001$, $58 \%$ shared variance): this is only $8 \%$ less than the variance in preference explained by the WAVE, and the association is not significantly weaker $(p=.85)$. For males, the relationship between the number of objects and color preference ( $r=-.67, p<.001,45 \%$ shared variance) is significantly weaker than that between the WAVE and color preference $(p<.005)$. However, for females, the relationship 
Fig. 1 Mean color preference ratings $( \pm 1 S E)$ and weighted affective valence estimates (WAVEs) for "saturated" (S), "light" (L), and "dark" (D) versions of eight hues ( $x$-axis: red, orange, yellow, chartreuse, green, cyan, blue, and purple), for all participants (top), for males (middle), and for females (bottom)

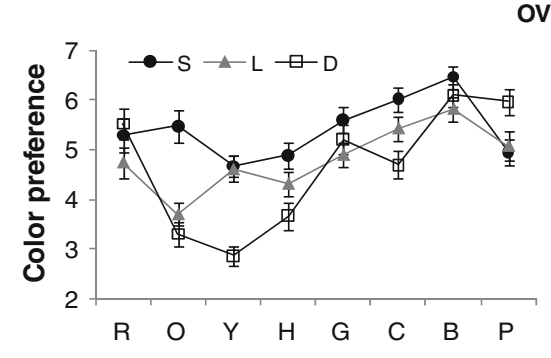

OVERALL

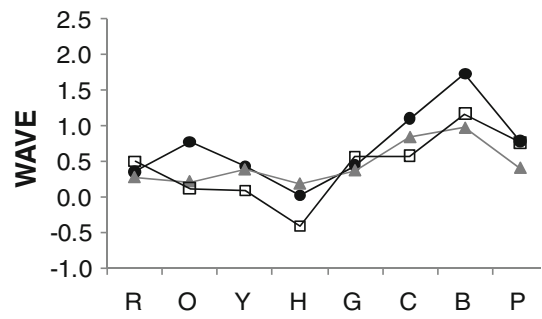

MALE
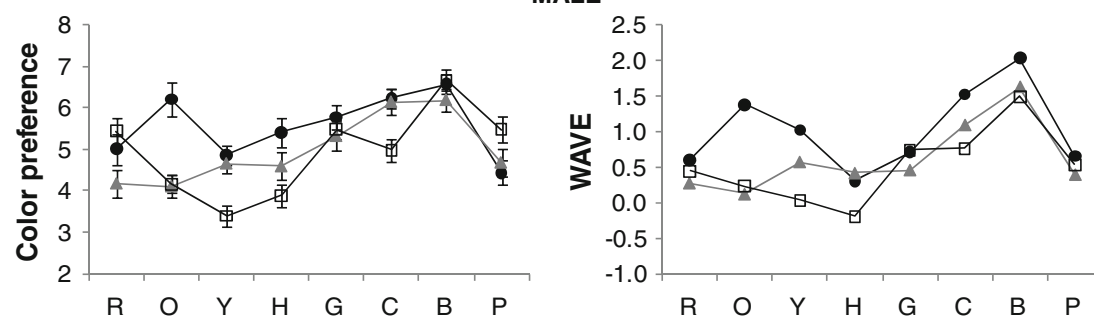

FEMALE
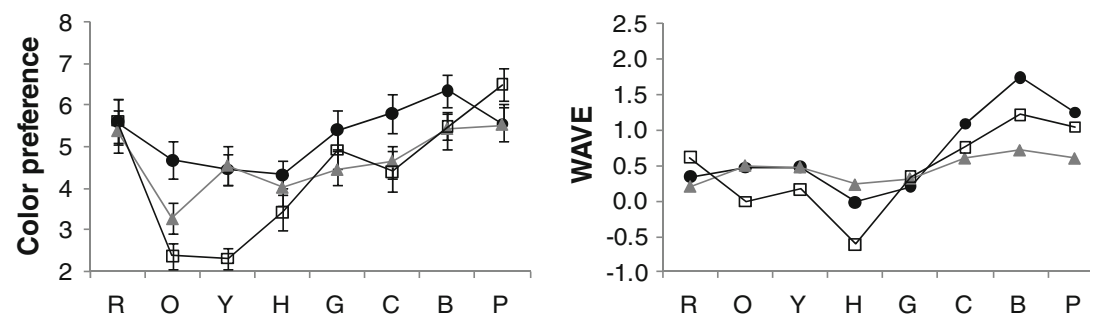

between the number of objects and color preference $(r=-.59$, $p<.005,35 \%$ shared variance) is not significantly weaker than that between the WAVE and color preference $(p=.21)$. There was no significant relationship between the number of objects associated with a color and the total object valence score for that color (all unweighted object valence ratings for a color summed) for the overall sample $(r=-.30, p=.15)$, for males $(r=-.37, p=.08)$, or for females $(r=-.27, p=.20)$.

To investigate the contribution of the number of objects to the positive association between the WAVE and color preference, we took this component out of the WAVE so that, rather than averaging the weighted object valence by the number of objects, the weighted object valences were

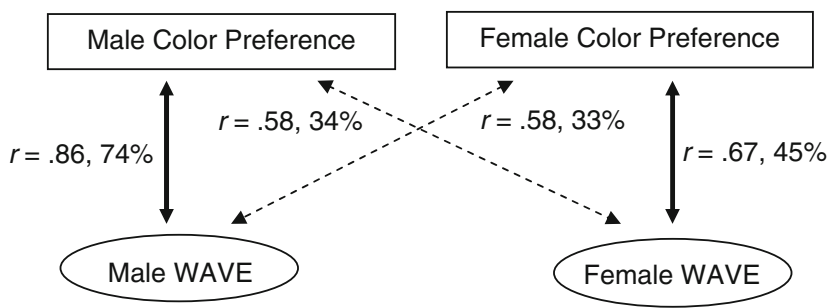

Fig. 2 The correlation coefficients and percentages of shared variance between color preference and weighted affective valence estimates (WAVEs) obtained from intrasex (solid lines) and intersex (dashed lines) preference-WAVE correlations summed (WAVE total ). Overall, this measure explained $38 \%$ of the variance in color preference $(r=.61, p<.005), 54 \%$ for male WAVE $\mathrm{Wtal}_{\text {tot }}$ and color preference $(r=.73, p<.001)$, and $33 \%$ for female WAVE $\mathrm{total}_{\text {an }}$ and color preference $(r=.58$, $p<.005)$. The variance explained by $\mathrm{WAVE}_{\text {total }}$ was significantly less than that explained by the original WAVE for the overall sample $(28 \%$ less, $p<.001)$ and for males $(20 \%$ less, $p<.05)$, but not for females $(12 \%$ less, $p=.97)$.

\section{Discussion}

Overall, the association between the WAVE and color preference was weaker for our British sample (66\%) than for Palmer and Schloss's (2010) American sample $(80 \%){ }^{3}$ but stronger than their preliminary data from a Japanese sample (44\%). We found the association between the WAVE and color preference to be significantly weaker for females $(45 \%)$ than for males $(74 \%)$. There appears to be some degree of gender specificity of the WAVE, as the male WAVE was significantly more strongly associated with male color preferences than was the female WAVE. However, the

\footnotetext{
${ }^{3}$ The shared variance between preference and the WAVE for the American sample was $79 \%$ when the muted set was excluded (Palmer \& Schloss, personal communication, June 2011)
} 
Fig. 3 Average color preference ratings $( \pm 1 S E$; left) and numbers of objects associated with each color (right) for "saturated" (S), "light" (L), and "dark" (D) versions of eight hues ( $x$-axis: red, orange, yellow, chartreuse, green, cyan, $\underline{\text { blue, and }}$ purple), for all participants (top), for males (middle), and for females (bottom)

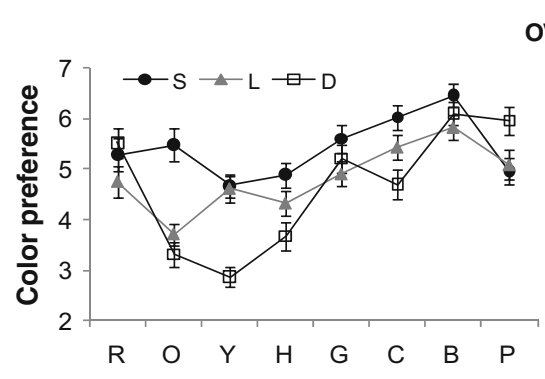

OVERALL
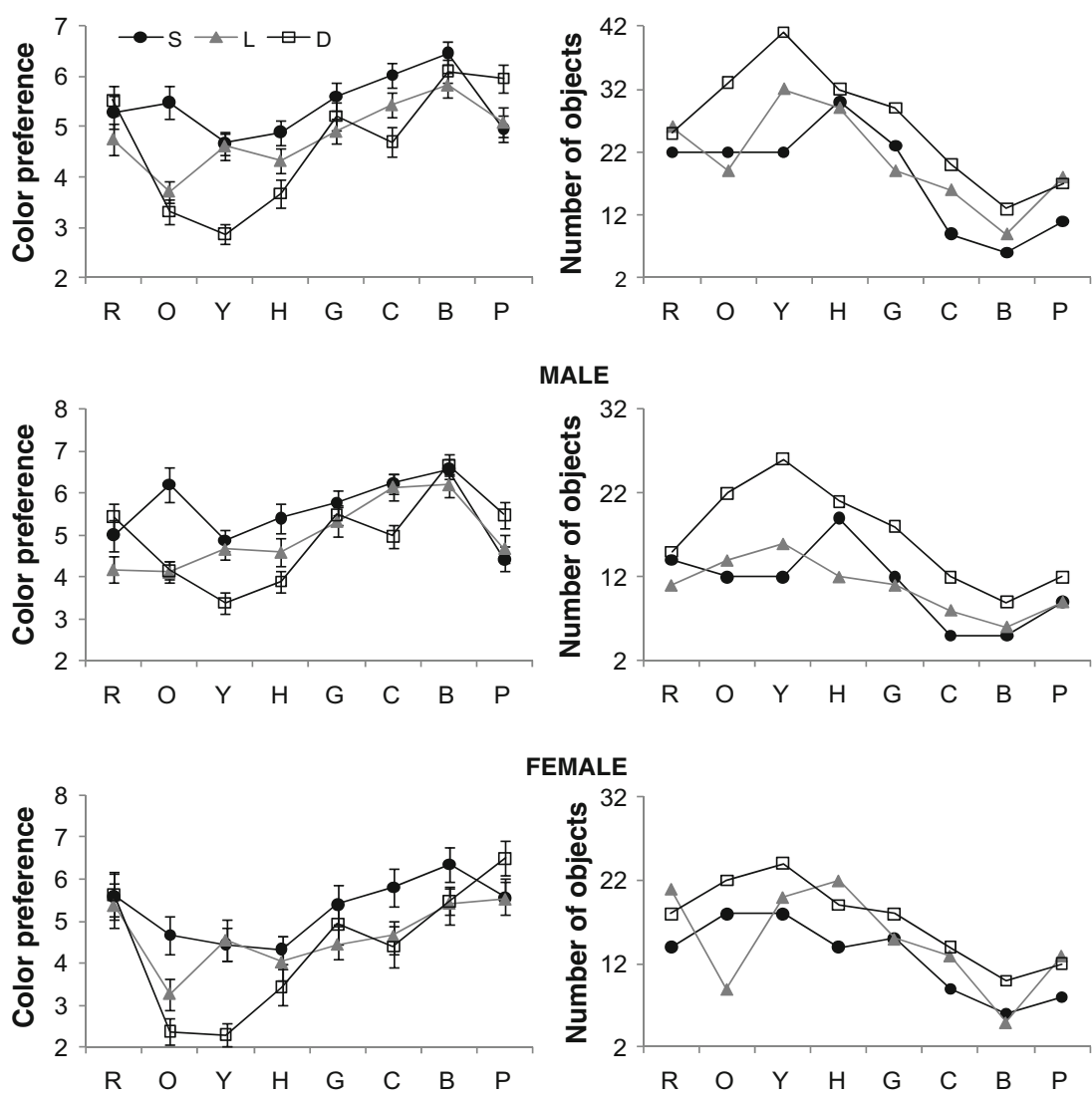

female WAVE was not significantly more strongly associated with female color preference than was the male WAVE, highlighting the limited ability of the female WAVE to capture female patterns of color preference.

One possible explanation for the sex difference revealed in our study is a difference in the relative importance of color associations for the concrete "public" objects that are measured by the WAVE versus abstract and/or personal objects that are not included in the WAVE. That is, male color preference may be strongly determined by color associations with concrete public objects, whereas female color preference may be more dependent on abstract and/or personal object associations than is male preference. Following Palmer and Schloss's (2010) procedure, we asked participants to not list objects whose color was personal to the participant (e.g., "my mum's sweater") or abstract concepts that are hard to validate (e.g., peace or love), so that object associations could be related to group color preferences. However, previous research has suggested that a broad range of "associative images" could be related to color preference (e.g., Saito, 1996), and a WAVE that fully captures the richness of color associations in everyday life might better explain female preferences and the preferences of certain cultures.

Palmer and Schloss (2010) proposed that the association between the WAVE and color preference is due to the average valence of color-associated objects causing or modifying color preference. However, our secondary analysis of their data, along with the data from the present investigation, has also established a relationship between the number of objects associated with a color and preference for that color. Colors associated with few objects are more likely to be liked than colors associated with many objects. The number of objects associated with a color did not directly measure object valence and was not significantly related to the total valence of objects associated with a color. ${ }^{4}$ For the overall sample and for females alone, the association between color preference and the number of objects was as strong as the association between overall and female color preference and the corresponding WAVEs.

This association between number of associated objects and color preference has implications for our understanding of what the WAVE measures. The WAVE averages the weighted object valences by the number of objects to give an intended measure of the average object valence for a color. However, as there is a negative relationship between the number of objects and preference, this serves to amplify the relationship between color preference and the WAVE.

\footnotetext{
${ }^{4}$ The number of objects was negatively associated with the WAVE $(r=-.82, p<.001)$, as would be expected, because the WAVE has the number of objects as a denominator.
} 
For example, averaging the weighted valences by the number of objects lowers the WAVE for colors with lots of objects associated with them (generally, disliked colors), relative to colors with few objects associated with them (generally, liked colors). One could also argue that it would be conceptually valid to sum the object valences for each color rather than to compute the average. For example, if lots of disliked objects are associated with a color, the associated object valence for that color should be lower than if only a few disliked objects are associated with the color. Summing object valences allows this, as adding lots of negative object ratings makes the total valence more negative. However, when object valences are summed for a color rather than averaged, the association between that WAVE and color preference is significantly weaker. This suggests that the success of the WAVE at accounting for color preferences is partly due to the negative relationship between color preference and the number of objects associated with a color.

Numerous explanations are possible for the negative association between the number of objects and color preference. One possible interpretation is that a color is liked because it is associated with few objects. The structure of the chromatic environment may mean that some object colors are more common than others. A color associated with few objects could have a stronger object identity, and therefore might elicit a clearer internal image than a color associated with multiple objects (e.g., blue is "sky," dark yellow is "a lot of stuff"). If this served to facilitate cognitive processing or codability, this could lead to greater preference, as research in other domains has established a link between ease of processing and preference (Winkielman \& Cacioppio, 2001). Alternatively, the association between the number of associated objects and color preference could indicate that few objects are associated with a color because we like it. The vocabulary used to describe negative stimuli is richer than that for positive stimuli, and negative stimuli elicit stronger, faster attentional responses than do positive or neutral stimuli, a phenomenon known at the "negativity bias" (see, e.g., Rozin \& Royzman, 2001). If this "negativity bias" affects colors, it could explain why more objects are listed for disliked colors. This would suggest that the valence of colors is affecting the object associations, with at least part of the causal relationship between object associations and color preference in the direction opposite to the one proposed by EVT.

In the present investigation, as in Palmer and Schloss (2010), different samples were tested on each task to avoid explicit or implicit cross-talk or priming between measures (i.e., if participants rate color preferences before listing color-object associations, preference ratings could prime participants explicitly or implicitly to think of more valence-congruent objects). By testing separate groups on each task, this potential bias was removed, and any conclusions about the relationship between color preference and color-object associations could be more clear-cut as a result. Relationships between these measures could not be revealed if different groups were highly variable in their color preferences or color-object associations. Here, the shared variance between randomly generated subgroups was consistently high across all measures for males and females (all $p \mathrm{~s}<.001$ ), and significant relationships between preference and color-object associations could be found. Relating the color preferences and color-object associations of different groups of participants is therefore methodologically sound, yet it should be borne in mind that the relationships revealed in the present study, and by Palmer and Schloss, are at the group level and are not necessarily present within an individual. The challenge for further research will be to explore these relationships on an individual level, but in such a way that potential explicit or implicit cross-talk between preference and object association tasks is prevented.

Our investigation has revealed several constraints of EVT in its current form. First, the average valence of color-associated objects relates less well to color preference for females than for males, at least for color associations with the concrete public objects measured here. Second, the average valence of color-associated objects accounts for color preference, in part because people generally like colors to the degree that they like the objects associated with those colors, but also because people's color preference is inversely related to the number of objects that they associate with a color. While there is some form of relationship between preference for a color and the objects associated with that color, further investigation of this relationship is clearly warranted.

Author note We thank Stephen Palmer and Karen Schloss for their generous and insightful discussion of color preference and for their comments on an earlier version of the manuscript.

\section{References}

Fletcher, R. (1981). City colour vision test. Windsor, U.K.: Keeler. Humphrey, N. (1976). The colour currency of nature. In T. Porter \& B. Mikellides (Eds.), Colour for Architecture. London: Studio-Vista.

Hurlbert, A. C., \& Ling, Y. (2007). Biological components of sex differences in color preference. Current Biology, 17, R623-R625. doi:10.1016/j.cub.2007.06.022

LoBue, V., \& DeLoache, J. S. (in press). Pretty in pink: The early development of gender-stereotyped colour preferences. British Journal of Developmental Psychology. doi:10.1111/j.2044835X.2011.02027.X

Ou, L.-C., Luo, M. R., Woodcock, A., \& Wright, A. (2004). A study of colour emotion and colour preference: Part III. 
Colour preference modelling. Color Research and Application, 29, 381-389.

Palmer, S. E., \& Schloss, K. B. (2010). An ecological valence theory of human color preference. Proceedings of the National Academy of Sciences, 107, 8877-8882. doi:10.1073/pnas.0906172107

Rozin, P., \& Royzman, E. B. (2001). Negativity bias, negativity dominance, and contagion. Personality and Social Psychology Review, 5, 296-320. doi:10.1207/S15327957PSPR0504_2

Saito, M. (1996). A comparative study of color preferences in Japan, China and Indonesia, with emphasis on the preference for white. Perceptual and Motor Skills, 83, 115-128.

Schloss, K. B., Poggesi, R. M., \& Palmer, S. E. (2011). Effects of university affiliation and "school spirit" on color preferences:
Berkeley versus Stanford. Psychonomic Bulletin \& Review, 18, 498-504. doi:10.3758/s13423-011-0073-1

Walton, W. E., Guilford, R. B., \& Guilford, J. P. (1933). Color preferences of 1,279 university students. The American Journal of Psychology, $45,322-328$

Winkielman, P., \& Cacioppo, J. T. (2001). Mind at ease puts a smile on the face: Psychophysiological evidence that processing facilitation elicits positive affect. Journal of Personality and Social Psychology, 81, 989-1000. doi:10.1037/00223514.81.6.989

Zajonc, R. B. (1980). Feeling and thinking: Preferences need no inferences. American Psychologist, 35, 151-175. doi:10.1037/ 0003-066X.35.2.151 